\title{
Elementary building blocks of graphene-nanoribbon-based electronic devices
}

\author{
Zhiping Xu and Quan-Shui Zheng \\ Department of Engineering Mechanics, Tsinghua University, Beijing 100084, China \\ Guanhua Chen \\ Department of Chemistry, The University of Hong Kong, Hong Kong, China
}

(Received 29 March 2007; accepted 9 May 2007; published online 1 June 2007)

\begin{abstract}
Graphene nanoribbon junction based electronic devices are proposed in this letter. Nonequilibrium Green's function calculations show that nanoribbon junctions tailored from single layer graphene with different edge shapes and widths can act as metal/semiconductor junctions and quantum dots can be implemented. In virtue of the possibilities of patterning monolayer graphene down to atomic precision, these structures, quite different from the previously reported two-dimensional bulk graphene or carbon nanotube devices, are expected to be used as the building blocks of the future nanoelectronics. (C) 2007 American Institute of Physics. [DOI: 10.1063/1.2745268]
\end{abstract}

Nanoelectronics, or molecular electronics, have been proposed as the alternative to silicon in future technical applications ${ }^{1}$ and have attracted great interest recently. In virtue of their unique structures and various functions, these nanostructures possess intriguing electromagnetic, mechanical, and optical features. Especially, carbon based nanostructures, such as fullerene, graphene, and carbon nanotubes, are the most interesting structures because of their rich variety of excellent physical properties. For instance, anomalous quantum Hall effects and massless Dirac electronic behavior have been discovered in the graphene systems, ${ }^{2,3}$ and these discoveries have sparked lots of investigations on this unique two-dimensional material. Tailored from monolayer graphene, graphene nanoribbon (GNR) with finite width has been shown to hold unusual electronic properties, ${ }^{4}$ depending on their edge shape and width. In more detail, graphene nanoribbons with zigzag edges (ZGNRs) possess spinpolarized peculiar edge states and spin-polarized electronic state provides half-metallicity under transverse electric field and has great potential in the application as spintronics. ${ }^{5}$ In contrast, the armchair edged nanoribbons (AGNRs) can be either metallic or semiconducting depending on their width. ${ }^{6}$ AGNR with width $N_{a}$ (named as $N_{a}$ AGNR in the conventional nomenclature) has been shown to be metallic only if $N_{a}=3 k+2$ and semiconducting otherwise, where $k$ is an integer.

From the experimental point of view, the fascinating feature of the ribbons is that the graphene material can be easily patterned using standard micro- or nanoelectronic lithography methods. Unlike the carbon nanotubes or other lowdimensional nanostructures, the GNRs with intricate submicrometer structures can now be fabricated, ${ }^{7-9}$ and it is believed that a combination of standard lithographic and chemical methods will help pattern the graphene with atomic precision down to the molecular level. The high mobility $\mu=2.7 \mathrm{~m}^{2} / \mathrm{V} \mathrm{s}$, large elastic mean free path $l_{e}=600 \mathrm{~nm}$, and phase coherence lengths $l_{\varphi}=1.1 \mu \mathrm{m}$ observed ${ }^{7}$ in the epitaxial graphene patterned suggest the use of pure GNR structures as the building blocks of the nanoscale confined and coherent electronic circuits. To realize the components such as field transistors ${ }^{9}$ and Coulomb blockade devices, experimentally controllable metal/semiconductor junctions and quantum dots will be essential. As proposed by Chico et al., ${ }^{10,11}$ these can be achieved by jointing different carbon nanotubes. However, the fabrication and control of the nanostructure of graphene ribbons are much more convenient than introducing pentagon-heptagon defects in carbon nanotubes as discussed. Therefore it is interesting to investigate the possibilities of ribbon junction based nanocircuits.

To this end, we have proposed several kinds of the GNRbased electronic devices in this letter. We show that, by controlling the tailoring process of GNRs with different edge shapes and widths, the metal/semiconductor junctions and quantum dots can be easily implemented experimentally. To validate this, electronic transport calculation using nonequilibrium Green's function method has been carried out following Landauer's approach. ${ }^{12}$ The electronic structure of the graphene lattice is described using the nearest-neighbor $\pi$-orbital tight-binding model and the hopping parameter $V_{p p \pi}=2.75 \mathrm{eV}$ is used. This simple topological model gives quantitative results compared with the local density approximation results except for the gap opening at small width as the consequence of the length changing of $\sigma$ bonds. ${ }^{6}$ By solving Green's function, the conductance was finally calculated as $G=G_{0} \operatorname{Tr}\left[\boldsymbol{\Gamma}_{L} \mathbf{G}^{R} \boldsymbol{\Gamma}_{R} \mathbf{G}^{A}\right]$ and the density of state is expressed as $D=-\operatorname{Im} \operatorname{Tr}\left[\mathbf{G}^{R}\right] / \pi^{11}$, where $G_{0}=2 e^{2} / h$ is the unit quanta of conductance including the spin degeneracy, $\mathbf{G}^{R(A)}$ is retarded (advanced) Green's function of the conductor, and $\boldsymbol{\Gamma}_{L(R)}$ is the spectral density describing the coupling between the left (right) lead and the conductor. In our model, the leads are represented using semi-infinite graphene ribbons attached to the conductor region, with the same shape and width.

First of all we investigate straight 11AGNR/10AGNR metal/semiconducting junction. The structure of the junction is considered by simply patching two different straight ribbons together, leave a width mismatch at the interface. The result shown in Fig. 1 indicates a gap $E_{g}=0.93 \mathrm{eV}$ near the Fermi energy and the imperfection at the interface induces a deviation of conductance from the steplike curve of the perfect ribbon. However, the van Hove singularities which are the characteristics of the one-dimensional (1D) system remain. 

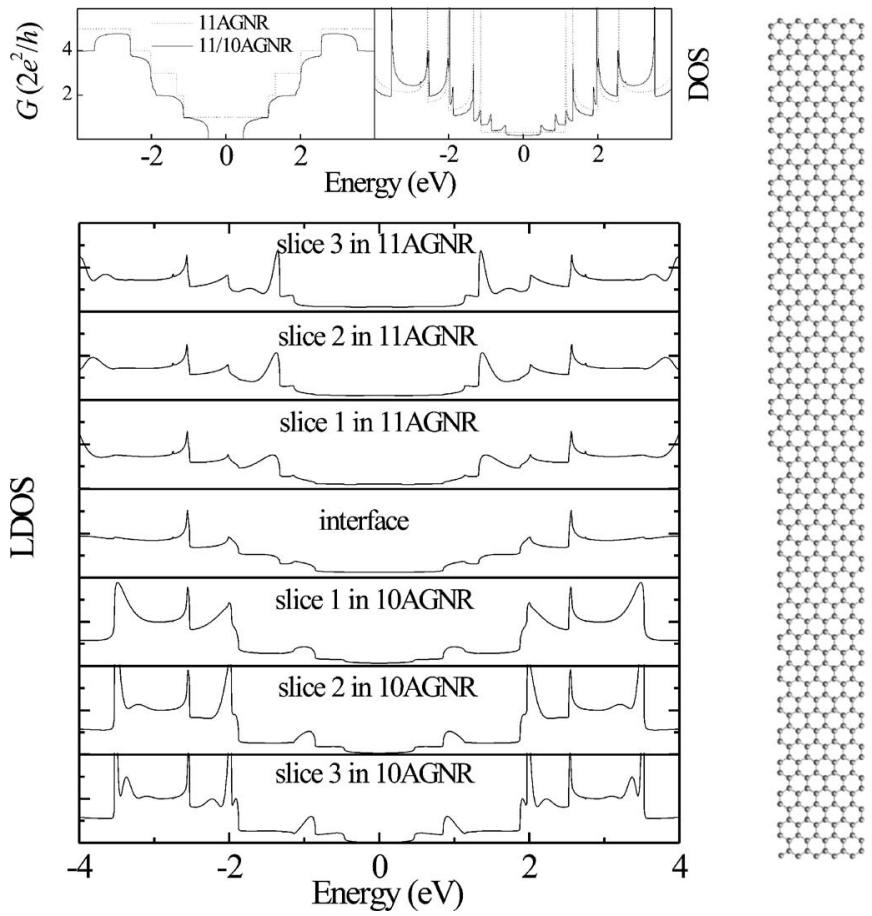

FIG. 1. 11AGNR/10AGNR metal/semiconducting junction: (Top) Conductance and DOS of the whole system. (Bottom) LDOS at slices near the interface. Slice $n(n=1,2$, and 3) represents the $n$th nearest slice to the interface and the vertical scale of DOS is 0.2 .

To examine the detailed electronic structure of the junction, a spatial-resolved localized density of states (LDOS) analysis is helpful. We have grouped the atoms into slices according to their distance from the interface. Each $4.26 \AA$ long slice (a unit cell of the perfect AGNR) in the 10AGNR, 11AGNR, and interface part contains 20, 22, and 21 atoms, respectively. The LDOS averaged at different slices are plotted in Fig. 1. From the semiconducting 10AGNR side we find that the LDOS is distorted near the interface and gap state appears through the contact with metallic 11AGNR. However, at slices far from the interface, at slice 3, for example, the perfect semiconducting behavior is mostly recovered. At the scattering interface the van Hove singularities have been smoothed and a 1D metallic structure gradually emerges as the distance from the interface increases from the 11AGNR side. The arising of the gap state near the interface characterizes the metal/semiconductor junction and suggests the possibilities of building Schottky devices.

Furthermore, L-shape GNR junctions with different orientations can be constructed. For instance, the LDOS of 8ZGNR/15AGNR junction with a $\pi / 6$ joint is analyzed in Fig. 2. As expected, the edge state of the 8ZGNR spreads into the semiconducting 15AGNR side. Because the ZGNR possesses a spin-polarized structure, this half-metal/ semiconducting junction inspires interests in the spintransport devices.

Besides the metal/semiconductor junction, the semiconductor/semiconductor junctions have also been investigated and defect states in the gap appear at the interface. Moreover in the ZGNR/ZGNR junctions, zero-conductance dips ${ }^{13}$ near the Fermi energy have been observed, caused by the complete backward scattering.

The metal/semiconductor junction also suggests quantum dot devices through combining two of them together. We now consider the 12AGNR/11AGNR/12AGNR junction. In
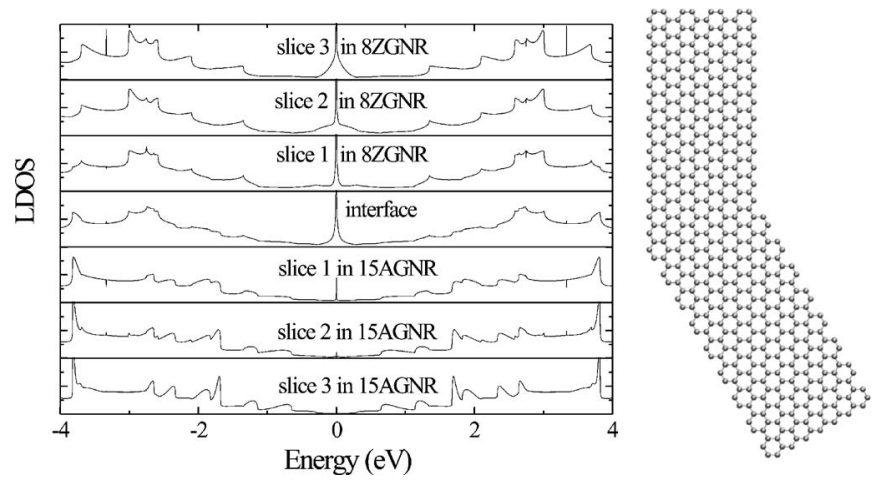

FIG. 2. Spatial-resolved LDOS in $8 Z$ GNR/15AGNR metal/semiconducting junction; the vertical scale of DOS is 0.2 .

this structure a central metallic ribbon is sandwiched by two semiconductor barriers where quantized states can be formed. Our calculation results depicted in Fig. 3 show two sharp DOS peaks inside the gap of semiconducting 12AGNR containing seven unit cells, with energies $E_{1,2}=0.2025$ and $-0.2025 \mathrm{eV}$. As seen from the spatial-resolved LDOS at $E$ $=0.2025$, the bounded state is localized inside the 11AGNR region. The structure of the quantum levels can be further tuned by changing the length of 11AGNR. From ou $r$ calculation, as it changes from one to eight unit cells, the energy spacing between the nearest peaks around the Fermi energy, i.e., $\Delta E=E_{1}-E_{2}$, gradually decreases from 0.785 to $0.385 \mathrm{eV}$, and their DOS becomes higher and sharper.

We have also observed quantized edge states within the 10AGNR/7ZGNR/10AGNR junctions by introducing two $\pi / 6$ joints. The results are shown in Fig. 3 where we find seven LDOS peaks inside the zero-conductance gap. The quantized states with $E=-0.3525,-0.1625,-0.05,0,0.05$, 0.1625 , and 0.3525 correspond to different LDOS patterns (see Fig. 4 for $E=0.3525$ ). The higher the energy, the more nodes the bounded standing wave has. The electron wave quantized pattern depends on the structure of the central region.

In conclusion, we have proposed nanoelectronic building blocks based on graphene nanoribbon junctions. Through tailoring GNRs into junctions of different edge shapes and

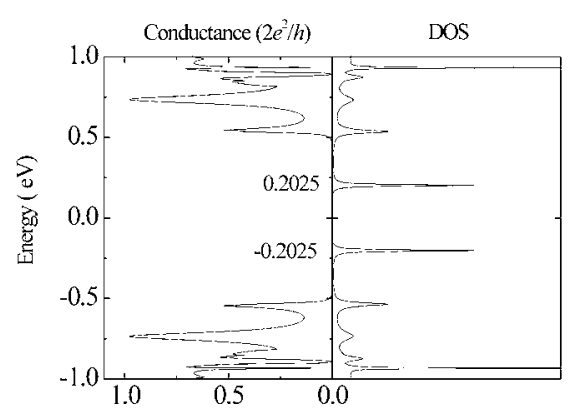

FIG. 3. Quantum dot structure based on 12AGNR/11AGNR/12AGNR junction: (Top) Conductance and DOS at low bias, where two isolated sharp peaks appear inside the gap. (Bottom) Spatial-resolved LDOS at $E$ $=0.2025 \mathrm{eV}$; the gray dot represents the ionic site and the radius of the circle around it corresponds to the value of LDOS. 

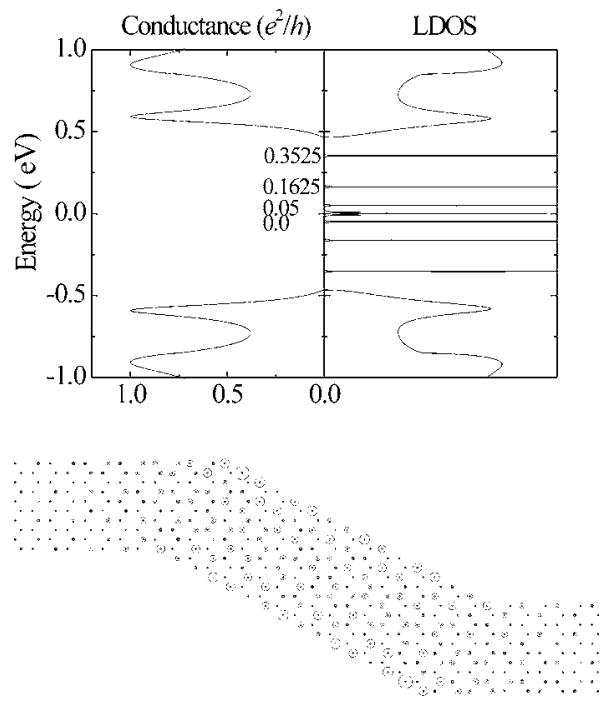

FIG. 4. Quantum dot structure based on 10AGNR/7ZGNR/10AGNR junction: (Top) Conductance and DOS. (Bottom) Spatial-resolved LDOS at $E=0.3525 \mathrm{eV}$.

widths, we can implement metal/semiconductor junctions and quantum dots in principle. In virtue of the possibility of molecular level patterning based on lithography and chemical methods, these devices are expected to be easier to fabricate in comparison with other structures such as the single molecule or carbon nanotube junctions and are expected to find great applications in the large-scale integrated nanocircuits in the future.

From an engineering point of view, the performance of GNR-based devices can be significantly affected by disorders arising from defects, substrate, and environmental temperature. ${ }^{14}$ Although the mean free path of electrons in graphene can exceed hundreds of nanometers at room temperature, ${ }^{7}$ the electron-phonon scattering was expected to contribute much in GNR with finite width. ${ }^{15}$ Thus besides the successful fabrication of perfect atomic structures, their stability under the operating environment and temperature should also be maintained to achieve real-world devices.

Although the method used in this work can reveal characteristic properties of the devices, it excludes the possibilities for describing current-voltage characteristics and time dependence. To elucidate these issues, time-dependent density-functional theory ${ }^{16}$ studies should be carried out. Very recently after this work has been finished, we notice that studies on the intrinsic current-voltage characteristics of GNR-based field effect transistor were reported, ${ }^{17}$ where very high level performance with on/off ratio up to $10^{4}$ was estimated. These results confirm the merits of GNR-based electronic devices and their potential as building blocks in nanoelectronics. ${ }^{18}$

The work is supported by the National Science Foundation of China through Grant Nos. 10172051, 10252001, and 10332020 and the Hong Kong Research Grant Council (NSFC/RGC Nos. HKU 764/05 and HKU 7012/04P). One of the authors (Z.X.) thanks Wenhui Duan, Tao Zhou, and Haiyun Qian for their help on the calculation methods.

${ }^{1}$ N. J. Tao, Nature Nanotechnology 1 173-181 (2006).

${ }^{2}$ K. S. Novoselov, A. K. Geim, S. V. Morozov, D. Jiang, M. I. Katsnelson, I. V. Grigorieva, S. V. Dubonos, and A. A. Firsov, Nature (London) 438, 197 (2005).

${ }^{3}$ Y. Zhang, Y. Tan, H. L. Stormer, and P. Kim, Nature (London) 438, 201 (2005).

${ }^{4}$ Y. Kobayashi, K. Fukui, T. Enoki, K. Kusakabe, and Y. Kaburagi, Phys. Rev. B 71, 193406 (2005).

${ }^{5}$ Y. Son, M. L. Cohen, and S. G. Louie, Nature (London) 444, 347 (2006).

${ }^{6}$ Y. Son, M. L. Cohen, and S. G. Louie, Phys. Rev. Lett. 97, 216803 (2006).

${ }^{7}$ C. Berger, Z. Song, X. Li, X. Wu, N. Brown, C. Naud, D. Mayou, T. Li, J. Hass, A. N. Marchenkov, E. H. Conrad, P. N. First, and W. A. de Heer, Science 312, 119 (2006).

${ }^{8}$ S. Liu, F. Zhou, A. Jin, H. Yang, Y. Ma, H. Li, C. Gu, L. Lu, B. Jiang, Q. Zheng, S. Wang, and L. Peng, Acta Phys. Sin. 54, 4251 (2005).

${ }^{9}$ Z. Chen, Y. Lin, M. Rooks, and P. Avouris, e-print cond-mat/0701599.

${ }^{10}$ L. Chico, V. H. Crespi, L. X. Benedict, S. G. Louie, and M. L. Cohen, Phys. Rev. Lett. 76, 971 (1996).

${ }^{11}$ L. Chico, M. P. Lopez Sancho, and M. C. Munoz, Phys. Rev. Lett. 81, 1278 (1998).

${ }^{12}$ J. Lu, J. Wu, W. Duan, F. Liu, B. Zhu, and B. Gu, Phys. Rev. Lett. 90, 156601 (2003).

${ }^{13}$ K. Wakabayashi, Phys. Rev. B 64, 125428 (2001).

${ }^{14}$ P. M. Ostrovsky, I. V. Gornyi, and A. D. Mirlin, Phys. Rev. B 74, 235443 (2006).

${ }^{15}$ D. Gunlycke, H. M. Lawler, and C. T. White, Phys. Rev. B 75, 085418 (2007).

${ }^{16}$ X. Zheng, F. Wang, C. Y. Yam, Y. Mo, and Guanhua Chen, e-print quantph/0702249.

${ }^{17}$ Q. Yan, B. Huang, J. Yu, F. Zheng, J. Zhang, J. Wu, B. Gu, F. Liu, and W. Duan, Nano Lett. (to be published).

${ }^{18}$ M. Ezawa, Phys. Status Solidi C 4, 489 (2007). 
Applied Physics Letters is copyrighted by the American Institute of Physics (AIP). Redistribution of journal material is subject to the AIP online journal license and/or AIP copyright. For more information, see http://ojps.aip.org/aplo/aplcr.jsp 